\title{
OPTIMAL REPRESENTATIONS IN MULTITREE DICTIONARIES WITH APPLICATION TO COMPRESSION.
}

\author{
Y. Huang ${ }^{\dagger}$, I. Pollak ${ }^{\dagger}$, M.N. Do ${ }^{\ddagger}$, and C.A. Bouman ${ }^{\dagger}$ \\ $\dagger$ Purdue University \\ School of ECE \\ West Lafayette, IN 47907 \\ $\ddagger$ University of Illinois at Urbana-Champaign \\ Department of ECE \\ Urbana, IL 61801
}

\begin{abstract}
We generalize our results of $[8,9]$ and propose a new framework of multitree dictionaries which include many previously proposed dictionaries as well as many new, very large, treestructured dictionaries. We present an efficient, globally optimal algorithm to find the best tree in such a dictionary. We describe a novel block image coder based on our framework, which is an improvement over our image coder presented in [8].
\end{abstract}

\section{INTRODUCTION.}

This paper focuses on optimal representation problems: given a signal, a dictionary of representations, and a cost function, the aim is to select the representation from the dictionary which minimizes the cost for the given signal.

The original papers on best basis search $[2,3]$ considered the wavelet packet bases [2] and bases of local cosines [12] on dyadic intervals. The bases in these dictionaries can be organized using a single tree: a binary tree in $1 \mathrm{D}$ and a quadtree in 2D. This organization was exploited in $[2,3]$ to devise a fast recursive tree pruning algorithm to find the best basis for any additive cost function. Since then, a number of efforts have sought to lift the restrictions that a fixed binary/quadtree structure imposes on the underlying dictionary $[1,5-9,11,20]$.

We propose a new framework of multitree dictionaries which include many previously proposed dictionaries as well as many new, very large, tree-structured dictionaries. Section 2 presents a globally optimal best basis search algorithm which is efficient, despite the size of these new dictionaries. We demonstrate the potential of these dictionaries in image compression by evaluating, in Section 3, our novel variable-block-size block-DCT image coder which optimally selects block sizes and shapes. (A preliminary version of this coder was presented in [8].) We show that this flexibility results in lower bit rates than either JPEG or the quadtree-based algorithm of [17], and that our coder is competitive when compared to the state-ofthe-art embedded wavelet coders such as JPEG2000 [19] and SPIHT [18]. Note, however, that our block-DCT coder has

This work was supported in part by a National Science Foundation (NSF) CAREER award CCR-0093105, an NSF grant IIS-0329156, a Purdue Research Foundation grant, and an NSF CAREER award CCR-0237633.

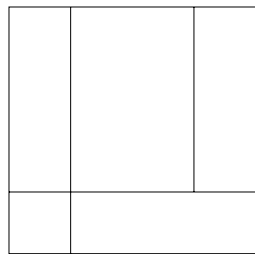

(a) A tiling.

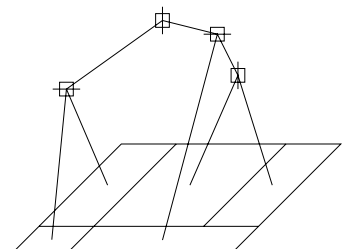

(b) A sequence of splits.
Fig. 1: An illustration of rectangular image tilings: (a) a tiling; (b) a corresponding sequence of splits. A short vertical (horizontal) line through a tree node represents a vertical (horizontal) split.

lower memory requirements (via processing block by block), and therefore may be more suitable in certain applications.

\section{MULTITREE DICTIONARIES.}

Multitree dictionaries are defined using the formalism of grammars [13]. We define a grammar $G=(A, S)$ to be a pair of the following sets: a set $A$ of symbols, ${ }^{1}$ and a set $S$ of allowed splits, also called productions, of the form $a \rightarrow \alpha$ where $a \in A$, and $\alpha$ is a finite sequence of elements of $A$. For example, the symbols may represent different rectangular tiles of an image domain, as in Fig. 1, or different bases in a dictionary. By starting with a single element of $A$, we can generate various sequences of elements of $A$ via recursive splitting-i.e., recursive application of productions, see Fig. 1. This process can be visualized as a tree where each production $a \rightarrow \alpha$ is depicted as a node labeled $a$ whose children are labeled with the elements of $\alpha$, left to right. We let a multitree dictionary $T_{a}(G)$ be the set of all such trees that can be produced by the gram$\operatorname{mar} G$, starting with the root symbol $a$. We say that a grammar $G=(A, S)$ is finite-depth if, for every $a \in A, T_{a}(G)$ is a finite set. This can be insured by only allowing a finite set of symbols to be descendants of $a$, and not allowing $a$ to be its own descendant.

Suppose that each symbol $u \in A$ is assigned a cost $c(u)$, that each production $u \rightarrow \alpha \in S$ is assigned a cost $\bar{c}(u \rightarrow \alpha)$, and that $\operatorname{cosT}(t)$ for any tree $t \in T_{a}(G)$ is the sum of the

\footnotetext{
${ }^{1}$ This is somewhat different from standard treatments of grammars [13] which distinguish between the start symbol which can only appear at the root, the nonterminal symbols which can only appear at the nonroot internal nodes, and terminal symbols which can only appear at the leaves. We, on the other hand, assume that any symbol in $A$ can appear at the root or any internal nodes or leaf nodes.
} 


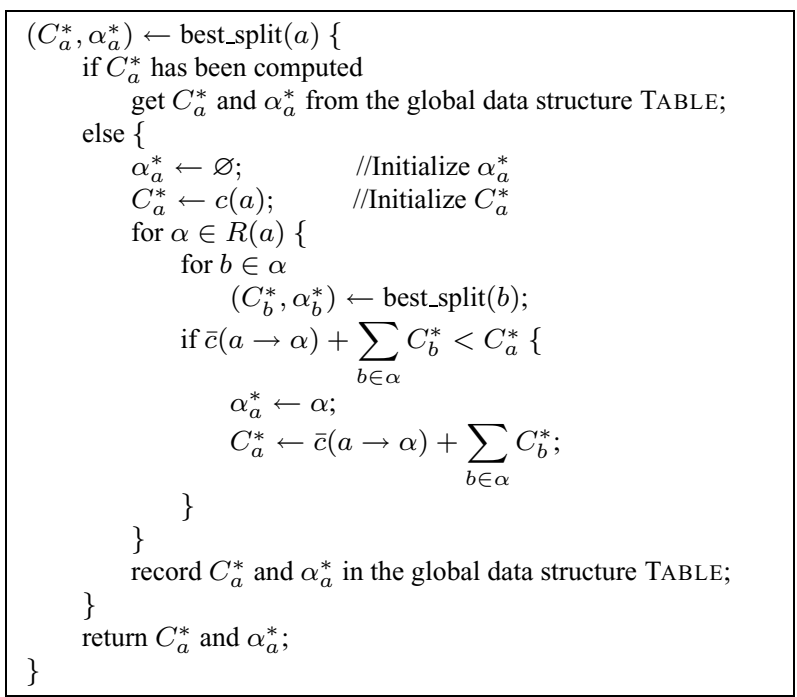

(a) Recursive calculation of best splits and costs.

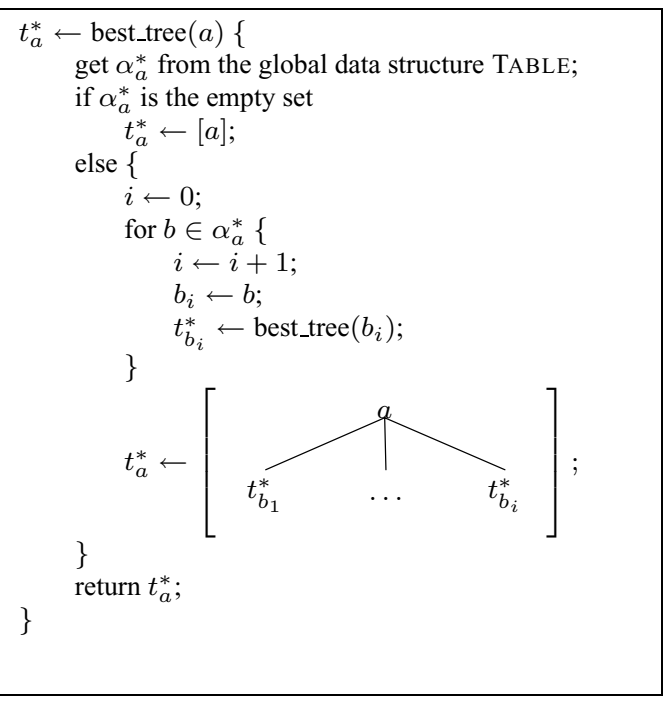

(b) Recursive generation of best tree.

Fig. 2: Pseudocode for the recursive calculation of the best splits and best costs, and for the generation of the best tree.

individual costs of all the productions comprising $t$, plus the sum of the costs of all its leaves:

$$
\operatorname{cosT}(t)=\sum_{u \rightarrow \alpha \in t} \bar{c}(u \rightarrow \alpha)+\sum_{u \in \text { leaves }(t)} c(u) .
$$

We would like to find the best tree in the dictionary $T_{a}(G)$, i.e., the tree $t_{a}^{*}$ whose cost is the smallest:

$$
t_{a}^{*}=\arg \min _{t \in T_{a}(G)} \cos \mathrm{T}(t) .
$$

We let $C_{a}^{*}$ be the corresponding cost: $C_{a}^{*}=\operatorname{cosT}\left(t_{a}^{*}\right)$. To illustrate our fast recursive algorithm for best tree search, we first suppose that the only allowed split of the symbol $a$ is: $a \rightarrow b_{1} b_{2}$. There is a tree $[a]$ in the multitree dictionary $T_{a}(G)$ which consists of one node labeled $a$, with $\operatorname{CosT}([a])=c(a)$. For any other tree $t \in T_{a}(G)$, its left subtree $t_{\text {left }}$ is in $T_{b_{1}}(G)$, and its right subtree $t_{\text {right }}$ is in $T_{b_{2}}(G)$. Therefore, since the cost is additive, $\operatorname{cosT}(t)=\bar{c}\left(a \rightarrow b_{1} b_{2}\right)+\operatorname{cosT}\left(t_{\text {left }}\right)+$ $\operatorname{cosT}\left(t_{\text {right }}\right)$. Consequently, the optimal tree is:

$$
t_{a}^{*}=\left\{\begin{array}{l}
{\left[t_{t_{b_{1}}^{*}}\right.} \\
{[a]}
\end{array}\right.
$$

In other words, we find the best trees $t_{b_{1}}^{*}$ and $t_{b_{2}}^{*}$ in the dictionaries $T_{b_{1}}(G)$ and $T_{b_{2}}(G)$, respectively, and compare their total cost plus the cost of the root production $a \rightarrow b_{1} b_{2}$, with the cost of the tree $[a]$.

We have a similar recursion in the general case. Let $R(a)$ be the set of the right-hand sides of all the productions that start with " $a \rightarrow$." Then one possible candidate for $t_{a}^{*}$ is $[a]$, with $\operatorname{cost} c(a)$, and all the other candidates are of the form:

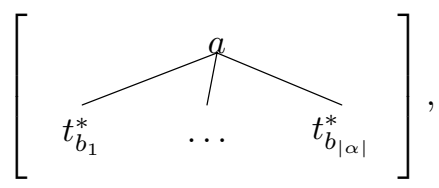

with cost $\bar{c}(a \rightarrow \alpha)+\sum_{i=1}^{|\alpha|} C_{b_{i}}^{*}$, for any $\alpha=\left(b_{1} \ldots b_{|\alpha|}\right) \in$ $R(a)$. To find the globally optimal $t_{a}^{*}$, we recursively search over these possibilities, terminating when $R(a)=\varnothing$ : in this case, $t_{a}^{*}=[a]$. The termination is guaranteed to happen in a finite number of steps for a finite-depth grammar. To avoid repetitive calculation, we store the optimal costs and corresponding productions in a global data structure called TABLE, as illustrated in the pseudocode of Fig. 2(a). Once this recursive call is done, the best tree can be generated from TABLE using the pseudocode in Fig. 2(b).

Note that standard wavelet packet and dyadic local cosine dictionaries [2,3], as well as anisotropic 2D wavelet packet dictionaries $[1,5]$, are all multitree dictionaries. Our algorithm, however, can also be used for a variety of other dictionaries, such as, for example, arbitrary dictionaries of block or lapped [12] bases in two or more dimensions. We emphasize that, despite its appearance, our fast recursive search algorithm for the globally optimal tree is neither a greedy search nor an exhaustive search algorithm. In fact, in many applications of interest where such large dictionaries can be used, our algorithm is guaranteed to find the optimal representation with only a modest increase in computation compared to the standard quadtree and dyadic algorithms.

\section{APPLICATION: A NEW BLOCK IMAGE CODER.}

To illustrate the potential of our framework for compression applications, we have developed a block discrete cosine transform (DCT) coder which operates on $16 \times 16$ image blocks. We use our search algorithm to optimally tile each block with rectangular subblocks; this tiling is optimized with respect to a rate-distortion cost [17]. If several different quantizers can be used for each subblock, the same algorithm can also be used to choose the optimal set of quantizers. Our algorithm encodes the quantized DCT coefficients for each subblock as well as 

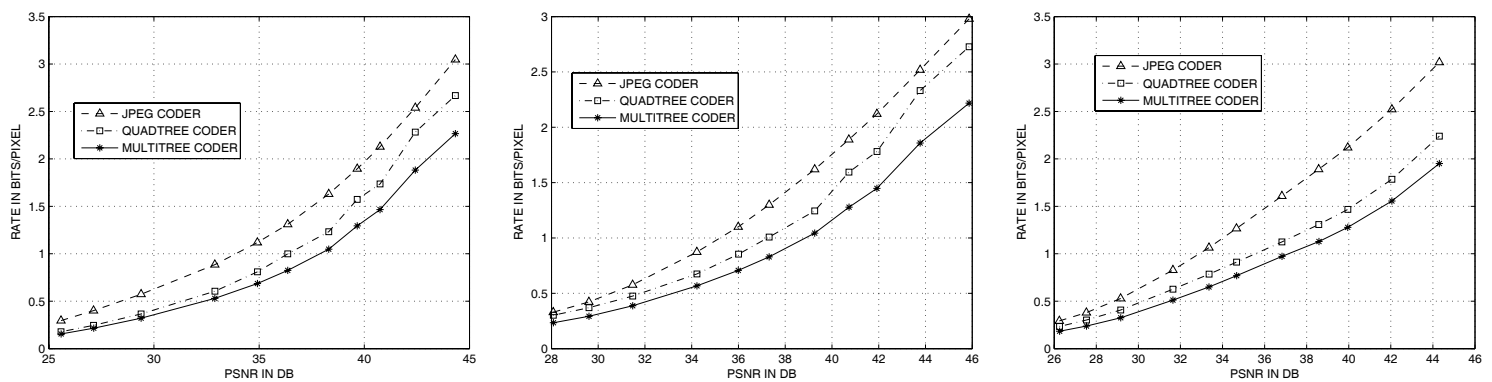

Fig. 3: Rate-distortion curves for "Barbara" (left), "Lena" (center), and "cameraman" (right).
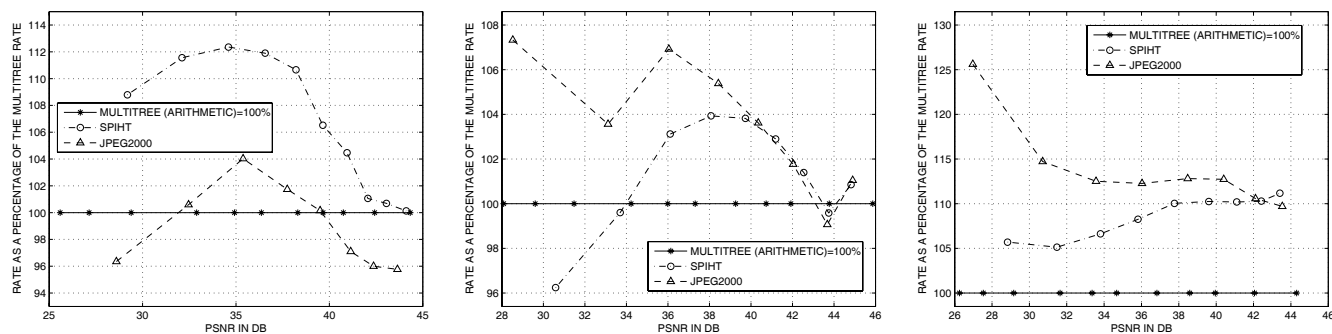

Fig. 4: Rate-distortion curves for "Barbara" (left), "Lena" (center), and "cameraman" (right). Bit rates are shown as percentages of the bit rate for the multitree algorithm with arithmetic coding.

the tree structure which corresponds to the optimal tiling.

Fig. 3 compares our compression algorithm ${ }^{2}$ with two other block-DCT coders: the standard JPEG and the quadtree-based algorithm of [17]. For our algorithm, we consider all rectangular subblocks with sides $4,8,12$, and 16 . This means that, for each $16 \times 16$ block, our fast recursive algorithm finds the best tiling from a collection of 68480 distinct tilings - this is in contrast to the quadtree method which only allows 17 distinct tilings, and the standard JPEG which only considers one tiling, namely, into $8 \times 8$ blocks. While the number of possible tilings for our method is drastically larger, the number of distinct possible subblocks in each block-which is what determines the computational complexity of our algorithm - is only 100 , compared to 21 for the quadtree method and 4 for the standard JPEG. We are thus able to search over a much larger set with only a modest increase in the computational burden. This leads to significant improvements in the compression performance, despite the fact that our algorithm requires more bits to encode the side information, i.e., the structure of the optimal tiling.

Fig. 3 shows rate-distortion curves for three images, "Barbara," "Lena," and "cameraman," at many bit rates and distortions. For each of these quadtree and multitree experiments, a target distortion was fixed, and the rate was minimized. Note that our multitree algorithm (solid) outperforms the standard JPEG (dash) by about 2-4 $\mathrm{dB}$ and the quadtree algorithm (dashdot) by about $1-2 \mathrm{~dB}$ at a fixed bit rate. Equivalently, the multitree algorithm represents compression savings of about 25$40 \%$ over the standard JPEG and $10-20 \%$ over the quadtree algorithm, for a fixed distortion. In these multitree experiments, the coefficients are Huffman coded using a procedure adapted from the baseline JPEG [15]. The use of arithmetic

\footnotetext{
${ }^{2} \mathrm{~A}$ detailed description of our image coder is given in [10]
}

coding instead of Huffman coding typically produces additional 5-8\% compression savings in our current experiments, and makes our algorithm competitive when compared to the state-of-the-art embedded wavelet coders such as JPEG2000 [19] and SPIHT [18] (both of which also use arithmetic coding). Fig. 4 shows the rate-distortion curves for JPEG2000, SPIHT, and our multitree coder, with the bit rates displayed as percentages of the multitree bit rate. For "cameraman" (right), our algorithm outperforms both JPEG2000 and SPIHT. It also does better than SPIHT for "Barbara" (left) and better than JPEG2000 for "Lena" (center).

Fig. 5 illustrates the results for the "Barbara" image at the bit rate 0.49 bits per pixel. In addition to a higher signalto-noise ratio, it is clear from the figure that, compared to both JPEG and the quadtree algorithm, the multitree algorithm results in both less blocky renditions of homogeneous areas of the image, sharper edges, and less ringing and blockiness in the textured areas and around the edges. It also produces sharper and more reliable renditions of edges than JPEG2000 and SPIHT, and is better than SPIHT at reproducing the textured portions in the lower-center part of the image. Our algorithm's visual drawback is the presence of some blocking artifacts, which is not surprising since it is based on a block transform.

To help reduce blocking artifacts and improve the overall performance of our image coder, we plan to explore the use of lapped transforms [12] which have been shown to be effective for coding textured images [14], as well as other recently proposed transforms such as bandelets [16] and contourlets [4]. We are also investigating several other ways of improving our image coder, such as alternative quantization strategies, improved ways of entropy coding of the coefficients, and further reducing the computational complexity. Finally, we plan to apply our algorithm to block size selection in variable-block-size 


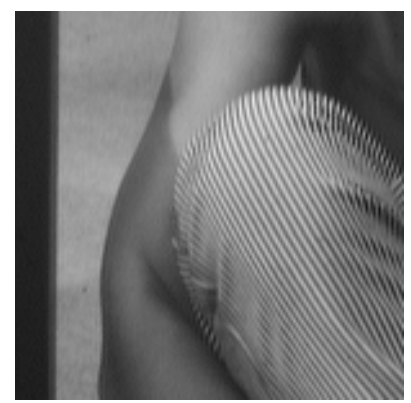

(a) A patch of "Barbara"

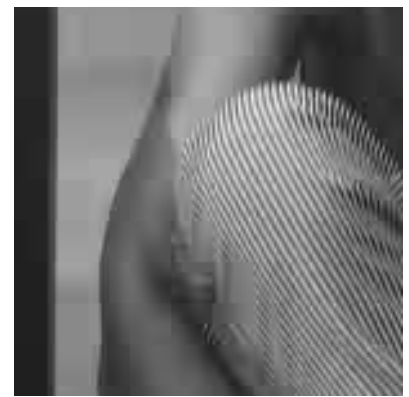

(d) Multitree

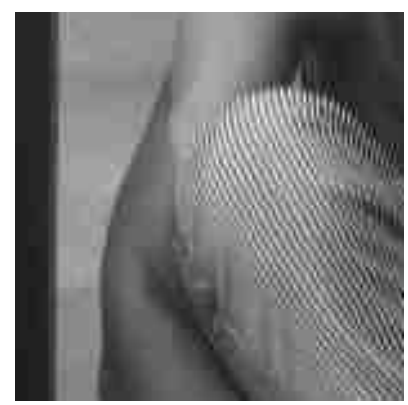

(b) JPEG

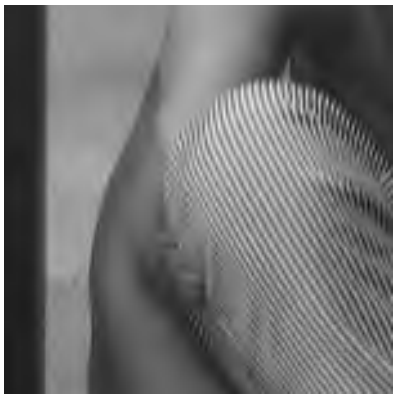

(e) JPEG2000

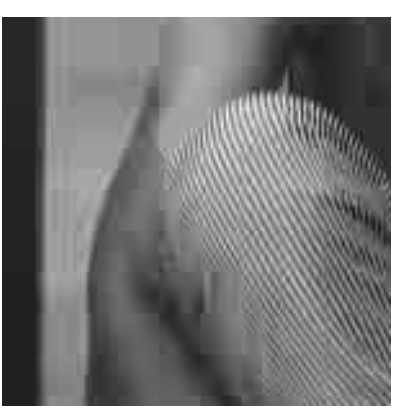

(c) Quadtree

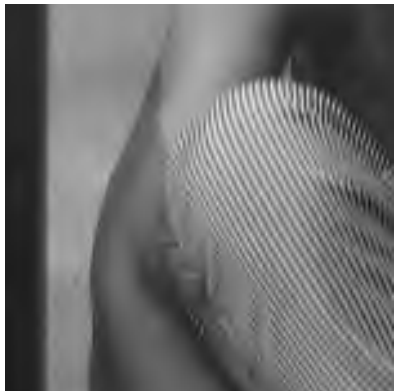

(f) SPIHT

Fig. 5: Results for the "Barbara" image at the bit rate of 0.49 bits per pixel: (a) a patch of the original image, (b) JPEG (PSNR for the overall image $=28.3 \mathrm{~dB})$, (c) quadtree [17] (PSNR = $30.5 \mathrm{~dB})$, (d) multitree (PSNR = $32.9 \mathrm{~dB})$, (e) JPEG2000 (PSNR = $32.8 \mathrm{~dB})$, and (f) SPIHT (PSNR = 32.0 dB).

video coders.

\section{REFERENCES}

[1] N. N. Bennett. Fast algorithm for best anisotropic Walsh bases and relatives. J. of Appl. and Comput. Harm. An., 8:86-103, 2000.

[2] R. R. Coifman, Y. Meyer, and M. V. Wickerhauser. Wavelet analysis and signal processing. In M. B. Ruskai et al., editor, Wavelets and Their Applications, pages 153-178. Jones and Bartlett, Boston, 1992.

[3] R. R. Coifman and M. V. Wickerhauser. Entropy based algorithms for best basis selection. IEEE Trans. Inf. Th, 38(2):713-718, 1992.

[4] M. N. Do and M. Vetterli. Contourlets. In G. V. Welland, editor, Beyond Wavelets. Academic Press, New York, 2003.

[5] D. L. Donoho. CART and best-ortho-basis: A connection. Ann. Stat., 25:1870-1911, 1997.

[6] C. Herley, J. Kovačević, K. Ramchandran, and M. Vetterli. Tilings of the time-frequency plane: construction of arbitrary orthogonal bases and fast tiling algorithms. IEEE Trans. Sig. Proc., 41(12):3341-3359, December 1993.

[7] C. Herley, Z. Xiong, K. Ramchandran, and M. T. Orchard. Joint spacefrequency segmentation using balanced wavelet packet tree for least-cost image representation. IEEE Trans. Im. Proc., 6(9):1213-1230, September 1997.

[8] Y. Huang, I. Pollak, and C. A. Bouman. Image compression with multitree tilings. In Proc. ICASSP-2005, Philadelphia, PA, March 2005.

[9] Y. Huang, I. Pollak, M. N. Do, and C. A. Bouman. Optimal tilings and best basis search in large dictionaries. In Proc. 37-th Asilomar Conf. Sig., Syst., and Comp., November 9-12 2003.

[10] Y. Huang, I. Pollak, M. N. Do, and C. A. Bouman. Fast search for best representations on multitree dictionaries. Technical Report TR-ECE-04-09, School of ECE, Purdue University, West Lafayette, IN 47907, December 2004. Submitted to IEEE Trans. Im. Proc.

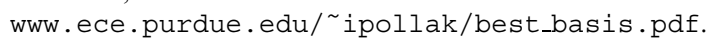

[11] M. Lindberg and L. F. Villemoes. Image compression with adaptive Haar-Walsh tilings. In Wavelet Applications in Signal and Image Processing VIII, Proc. SPIE 4119, 2000.

[12] H. Malvar. Signal Processing with Lapped Transforms. Artech House, 1992.
[13] C. Manning and H. Schütze. Foundations of Statistical Natural Language Processing. MIT Press, 1999.

[14] F. Meyer. Image compression with adaptive local cosines: A comparative study. IEEE Trans. Im. Proc., 11(6):616-629, June 2002.

[15] W. B. Pennebaker and J. L. Mitchell. JPEG Still Image Data Compression Standard. Van Nostrand Reinhold, New York, 1993.

[16] E. Le Pennec and S. G. Mallat. Sparse geometric image representations with bandelets. To appear in IEEE Trans. Im. Proc., 2005.

[17] K. Ramchandran and M. Vetterli. Best wavelet packet bases in a ratedistortion sense. IEEE Trans. Im. Proc., 2(2):160-175, Apr. 1993.

[18] A. Said and W. A. Pearlman. A new, fast, and efficient image codec based on set partitioning in hierarchical trees. IEEE Trans. Circ. Syst. Vid. Tech., 6(3):243-250, 1996.

[19] D. Taubman. High performance scalable image compression with EBCOT. IEEE Trans. Im. Proc., 9(7):1158-1170, July 2000.

[20] Z. Xiong, K. Ramchandran, C. Herley, and M. T. Orchard. Flexible treestructured signal expansions using time-varying wavelet packets. IEEE Trans. Sig. Proc., 45(2):333-345, February 1997. 